\title{
Spatial Pythagorean Hodograph Quintics and the Approximation of Pipe Surfaces
}

\author{
Zbyněk Šír and Bert Jüttler \\ Johannes Kepler University, Institute of Applied Geometry, \\ Altenberger Str. 69, 4040 Linz, Austria. \\ \{zbynek.sir, bert.juettler\}@jku.at, \\ WWW home page: http://www.ag.jku.at/
}

\begin{abstract}
As observed by Farouki et al. [9], any set of $C^{1}$ space boundary data (two points with associated first derivatives) can be interpolated by a Pythagorean hodograph $(\mathrm{PH})$ curve of degree 5 . In general there exists a two dimensional family of interpolants.

In this paper we study the properties of this family in more detail. We introduce a geometrically invariant parameterization of the family of interpolants. This parameterization is used to identify a particular solution, which has the following properties. Firstly, it preserves planarity, i.e., the interpolant to planar data is a planar PH curve. Secondly, it has the best possible approximation order (4). Thirdly, it is symmetric in the sense that the interpolant of the "reversed" set of boundary data is simply the "reversed" original interpolant. These observations lead to a fast and precise algorithm for converting any (possibly piecewise) analytical curve into a piecewise $\mathrm{PH}$ curve of degree 5 which is globally $C^{1}$.

Finally we exploit the rational frames associated with any space $\mathrm{PH}$ curve (Euler-Rodrigues frame) in order to obtain a simple rational approximation of pipe surfaces with a piecewise analytical spine curve and we analyze its approximation order.
\end{abstract}

\section{Introduction}

Pythagorean hodograph (PH) curves (see the survey [11] and the references cited therein), form a remarkable subclass of polynomial parametric curves. They have a piecewise polynomial arc length function and, in the planar case, rational offset curves. These curves provide an elegant solution of various difficult problems occurring in applications, in particular in the context of CNC (computer-numericalcontrol) machining.

In the planar case, the properties and various constructions of $\mathrm{PH}$ curves have been thoroughly studied, e.g., $[1,6,8,7,18,23]$. Due to the constrained nature of $\mathrm{PH}$ curves, all constructions - which are linear in the case of polynomial curves - become nonlinear in the PH case. Consequently, they may have more than one solution, and the problem of choosing the 'best' solution has to be addressed, e.g. by analyzing the approximation order or using the rotation index $[15,18$, $20-22]$. 
Spatial PH curves were introduced by Farouki and Sakkalis in 1994 [5], and they have later been characterized using results about Pythagorean quadruples in the ring of polynomials and quaternion calculus $[2,4,10]$. Spatial $\mathrm{PH}$ curves can be equipped with rational frames, which were studied in $[3,13,17]$.

Various constructions were also given, e.g. a global method for $C^{2}$ interpolation of point data by quintic splines has been presented in [12]. Hermite interpolation of $G^{1}$ boundary data was addressed in [17], and $C^{1}$ Hermite interpolation by $\mathrm{PH}$ quintics was discussed in [9]. In the latter case, the authors identify a family of interpolants to any $C^{1}$ Hermite data which depends on two free parameters, and a heuristic choice for them is given. Later, this has also been related to helical interpolants [14].

The present paper is devoted to the problem of $C^{1}$ Hermite interpolation by spatial $\mathrm{PH}$ quintics, and to the approximation of pipe surfaces and sweeping surfaces. We study the family of interpolants and identify the solution which has the best approximation order, preserves planarity, and is symmetric with respect to the reversion $t \mapsto(1-t)$ of the parameter interval $[0,1]$.

The remainder of the paper is organized as follows. First we recall some basic facts about quaternion algebra and $\mathrm{PH}$ curves. The first part of Section 3 summarizes the approach taken in [9] to the problem of $C^{1}$ Hermite interpolation by $\mathrm{PH}$ quintics. In the second part we introduce a parameterization of the family of interpolants with respect to a standard position. We prove that this parameterization is geometrically invariant and symmetric.

Section 4 provides a qualitative analysis of the solutions. We give an asymptotical analysis, including approximation order, and we identify the parameter values which preserve planarity. Based on these results, we use optimal solution for converting analytical curves into piecewise $\mathrm{PH}$ quintic curves and for the approximation of pipe surfaces. Finally we conclude the paper.

\section{Preliminaries}

In order to make this paper self-contained, we recall some basic facts about quaternions and Pythagorean Hodograph curves.

\subsection{Quaternions}

Quaternions (see e.g. [19] for an elementary introduction) are elements

$$
\mathcal{A}=a+a_{x} \mathbf{i}+a_{y} \mathbf{j}+a_{z} \mathbf{k}
$$

of 4-dimensional real linear space $\mathbb{Q}$ with basis $1, \mathbf{i}, \mathbf{j}, \mathbf{k}$. The space $\mathbb{Q}$ has the structure of a non-commutative field, where the multiplication is defined by the relations

$$
\mathbf{i}^{2}=\mathbf{j}^{2}=\mathbf{k}^{2}=\mathbf{i j k}=-1
$$

of the basis elements, which imply

$$
\mathbf{i j}=-\mathbf{j i}=\mathbf{k}, \quad \mathbf{j k}=-\mathbf{k j}=\mathbf{i}, \quad \mathbf{k i}=-\mathbf{i k}=\mathbf{j} .
$$


The conjugate of any quaternion (1) is defined as $\mathcal{A}^{*}=a-a_{x} \mathbf{i}-a_{y} \mathbf{j}-a_{z} \mathbf{k}$, and its absolute value is the non-negative real number

$$
|\mathcal{A}|^{2}=\sqrt{\mathcal{A} \mathcal{A}^{*}}=\sqrt{\mathcal{A}^{*} \mathcal{A}}=\sqrt{a^{2}+a_{x}^{2}+a_{y}^{2}+a_{z}^{2}} .
$$

Unit quaternions, which are characterized by $|\mathcal{A}|=1$, form a multiplicative group. Pure quaternions are distinguished by having a vanishing scalar part.

Quaternions are traditionally used in classical mechanics. Any vector $\mathbf{c}=$ $\left[c_{x}, c_{y}, c_{z}\right]^{\top} \in \mathbb{R}^{3}$ is identified with the pure quaternion $c_{x} \mathbf{i}+c_{y} \mathbf{j}+c_{z} \mathbf{k}$. Any unit quaternion $\mathcal{U}$ can be expressed in the form

$$
\mathcal{U}=\cos \frac{\theta}{2}+\mathbf{u} \sin \frac{\theta}{2}, \quad \theta \in[-\pi, \pi),
$$

where $\mathbf{u}$ is a unit pure quaternion. Then the mapping

$$
U: \mathbb{R}^{3} \rightarrow \mathbb{R}^{3}: U(\mathbf{c})=\mathcal{U} \mathbf{c} \mathcal{U}^{*}
$$

represents a rotation through the angle $\theta$ about the axis spanned by the direction vector $\mathbf{u}$.

In the sequel we will use the the abbreviation

$$
\mathcal{Q}(\phi)=(\cos \phi+\mathbf{i} \sin \phi)
$$

for unit quaternions with vanishing $\mathbf{j}$ and $\mathbf{k}$ components.

For the construction of $\mathrm{PH}$ Hermite interpolants which is described below, the following Lemma proved in [9, section 3.2] is essential.

Lemma 1. For a given pure quaternion $\mathbf{c}$, which is not a negative multiple of $\mathbf{i}$, all solutions of the equation

$$
\mathcal{A} \text { i } \mathcal{A}^{*}=\mathbf{c}
$$

are expressed as

$$
\mathcal{A}(\phi)=\sqrt{|\mathbf{c}|} \frac{\frac{\mathbf{c}}{|\mathbf{c}|}+\mathbf{i}}{\left|\frac{\mathbf{c}}{|\mathbf{c}|}+\mathbf{i}\right|} \mathcal{Q}(\phi), \quad \phi \in[0,2 \pi) .
$$

If $\mathbf{c}$ is a negative multiple of $\mathbf{i}$, then a suitable limit of the formula (9) must be taken.

\subsection{Pythagorean Hodograph curves}

The hodograph of a space curve $\mathbf{p}(t)=[x(t), y(t), z(t)]^{\top}$ of degree $n$ is the curve $\mathbf{h}(t)=\left[x^{\prime}(t), y^{\prime}(t), z^{\prime}(t)\right]^{\top}$ of degree $n-1$, where ' denotes the first derivative. Recall that a polynomial curve is called Pythagorean Hodograph (PH), if the length of its tangent vector depends in a (piecewise) polynomial way on the 
parameter. In particular $\mathbf{p}(t)=[x(t), y(t), z(t)]^{\top}$ is called space PH curve if there exists a polynomial $\sigma(t)$ such that

$$
x^{\prime}(t)^{2}+y^{\prime}(t)^{2}+z^{\prime}(t)^{2}=\sigma^{2}(t) .
$$

If $\operatorname{gcd}\left(x^{\prime}(t), y^{\prime}(t), z^{\prime}(t)\right)$ is a square, then equation (10) holds if and only if there exist polynomials $u(t), v(t), p(t), q(t)$ such that

$$
\begin{aligned}
x^{\prime}(t) & =u^{2}(t)+v^{2}(t)-p^{2}(t)-q^{2}(t) \\
y^{\prime}(t) & =2 u(t) q(t)+2 v(t) p(t) \\
z^{\prime}(t) & =2 v(t) q(t)-2 u(t) p(t) \\
\sigma(t) & =u^{2}(t)+v^{2}(t)+p^{2}(t)+q^{2}(t)
\end{aligned}
$$

see [4]. This result can be reformulated using quaternions [2, 10]. Any spatial polynomial curve $\mathbf{p}(t)=[x(t), y(t), y(t)]^{\top}$ is identified with the pure-quaternionvalued function $\mathbf{p}(t)=x(t) \mathbf{i}+y(t) \mathbf{j}+z(t) \mathbf{k}$. The $\mathrm{PH}$ curves are then characterized as follows.

Lemma 2. Let $\mathbf{p}(t)=x(t) \mathbf{i}+y(t) \mathbf{j}+z(t) \mathbf{k}$ be a space polynomial curve, such that $\operatorname{gcd}\left(x^{\prime}(t), y^{\prime}(t), z^{\prime}(t)\right)$ is the square of a polynomial ${ }^{1}$. Then $\mathbf{p}(t)$ is PH if and only if there exists a quaternion-valued polynomial $\mathcal{A}(t)=u(t)+v(t) \mathbf{i}+p(t) \mathbf{j}+q(t) \mathbf{k}$ such that

$$
\mathbf{h}(t)=\mathcal{A}(t) \mathbf{i} \mathcal{A}^{*}(t) .
$$

The arc length function of the PH curve is a polynomial obtained by integrating $|\mathcal{A}(t)|^{2}=\mathcal{A}(t) \mathcal{A}^{*}(t)$.

Consequently, the construction of a $\mathrm{PH}$ curve is reduced to the construction of a suitable curve $\mathcal{A}(t)$. This curve will be called the preimage.

\section{$3 \quad C^{1}$ Hermite interpolation by space quintics}

Following [9], we construct a spatial $\mathrm{PH}$ curve $\mathbf{p}(t)$ which matches given $C^{1}$ Hermite boundary data. More precisely, the curve is to interpolate the end points $\mathbf{p}_{0}, \mathbf{p}_{1}$ and the tangent vectors (or derivation vectors) $\mathbf{t}_{0}, \mathbf{t}_{1}$. The cases $\mathbf{t}_{0}=0$ or $\mathbf{t}_{1}=0$, and $\mathbf{t}_{0}=-\mathbf{t}_{1}$, which correspond to singular points at the segment end points, and to antiparallel tangent vectors of the same lengths, will be excluded.

\subsection{Construction of the interpolants}

Two curves $\mathbf{p}(t), \tilde{\mathbf{p}}(t)$ share the same hodograph if and only if they differ only by translation. Consequently a space $\mathrm{PH}$ curve $\mathbf{p}(t)$ is fully determined by the preimage $\mathcal{A}(t)$ and by the location of its starting point $\mathbf{p}(0)$.

The position of $\mathbf{p}_{0}$ can be matched by a suitable choice of the integration constant. The remaining $3 \cdot 3=9$ conditions must be satisfied by choosing the

\footnotetext{
${ }^{1}$ This includes the generic case $\operatorname{gcd}\left(x^{\prime}(t), y^{\prime}(t), z^{\prime}(t)\right)=1$.
} 
control points of the preimage $\mathcal{A}(t)$. Hence, the degree of $\mathcal{A}(t)$ has to be at least 2 , yielding $3 \cdot 4=12$ free parameters. As shown in [10], the representation preimage $\rightarrow$ hodograph (12) has one dimensional fibers. Therefore one can expect that there will be a two dimensional system of $\mathrm{PH}$ interpolants of degree $2 \cdot 2+1=5$.

We will use the Bernstein-Bézier representation [16] of the hodograph $\mathbf{h}(t)=$ $\mathbf{p}^{\prime}(t)$ and the preimage $\mathcal{A}(t)$ :

$$
\mathbf{h}(t)=\sum_{i=0}^{4} \mathbf{h}_{i} B_{i}^{4}(t), \quad \mathcal{A}(t)=\sum_{i=0}^{2} \mathcal{A}_{i} B_{i}^{2}(t), \quad t \in[0,1],
$$

where $\mathbf{h}_{i}$ (pure quaternions) and $\mathcal{A}_{i}$ (quaternions) are the control points and $B_{j}^{n}(t)=\left(\begin{array}{c}n \\ j\end{array}\right) t^{j}(1-t)^{n-j}$ are the Bernstein polynomials. The interpolation conditions lead to the equations

$$
\mathbf{h}_{0}=\mathbf{t}_{0}, \quad \mathbf{h}_{4}=\mathbf{t}_{1}, \text { and } \frac{1}{5} \sum_{i=0}^{4} \mathbf{h}_{i}=\left(\mathbf{p}_{1}-\mathbf{p}_{0}\right),
$$

which have to be satisfied by the control points of the hodograph. After expressing them in terms of the control points of the preimage curve, and a suitable re-arranging, one arrives at the following system of equations [9]:

$$
\mathcal{A}_{0} \mathbf{i} \mathcal{A}_{0}^{*}=\mathbf{t}_{0}, \quad \mathcal{A}_{2} \mathbf{i} \mathcal{A}_{2}^{*}=\mathbf{t}_{1},
$$

and

$$
\begin{aligned}
\left(3 \mathcal{A}_{0}+4 \mathcal{A}_{1}+3 \mathcal{A}_{2}\right) \mathbf{i}\left(3 \mathcal{A}_{0}+4 \mathcal{A}_{1}+3 \mathcal{A}_{2}\right)^{*} & = \\
& 120\left(\mathbf{p}_{1}-\mathbf{p}_{0}\right)-15\left(\mathbf{t}_{1}+\mathbf{t}_{0}\right)+5\left(\mathcal{A}_{0} \mathbf{i} \mathcal{A}_{2}^{*}+\mathcal{A}_{2} \mathbf{i} \mathcal{A}_{0}^{*}\right) .
\end{aligned}
$$

These three equations have the form (8). From (15) we get two 1 -parametric systems of solutions $\mathcal{A}_{0}\left(\phi_{0}\right)$ and $\mathcal{A}_{2}\left(\phi_{2}\right)$ of the form (9) (step 1). After substituting them into (16), we get (step 2) a 3-parametric system of solutions $\mathcal{A}_{1}\left(\phi_{0}, \phi_{1}, \phi_{2}\right)$.

Summing up, we arrive at a three-parametric system of suitable preimages

$$
\mathcal{A}(t)=\mathcal{A}_{0}\left(\phi_{0}\right) B_{0}^{2}(t)+\mathcal{A}_{1}\left(\phi_{0}, \phi_{1}, \phi_{2}\right) B_{1}^{2}(t)+\mathcal{A}_{2}\left(\phi_{2}\right) B_{2}^{2}(t) .
$$

However, as observed in [9], the resulting $\mathrm{PH}$ curve depends only on the differences of the angular parameters $\phi_{0}, \phi_{1}, \phi_{2}$, and therefore the preimages with a fixed value of $\phi_{1}$ still give all possible $\mathrm{PH}$ interpolants. We fix $\phi_{1}=0$ and denote the system of preimages as $\mathcal{A}_{\phi_{0}, \phi_{2}}(t){ }^{2}$

The quintic PH interpolants are obtained from

$$
\mathbf{p}_{\phi_{0}, \phi_{2}}(\tau)=\mathbf{p}_{0}+\int_{0}^{\tau} \mathcal{A}_{\phi_{0}, \phi_{2}}(t) \mathbf{i} \mathcal{A}_{\phi_{0}, \phi_{2}}^{*}(t) \mathrm{d} t
$$

As a first example, Figure 1 shows some representatives of the system of all $\mathrm{PH}$ quintic interpolants to the data

$$
\mathbf{p}_{0}=(0,0,0)^{\top}, \quad \mathbf{p}_{1}=(1,0,0)^{\top}, \quad \mathbf{t}_{0}=(3,3,0)^{\top}, \quad \mathbf{t}_{1}=(3,3,0)^{\top} .
$$

Note, that while this data are in fact planar (since it is contained in the $x y$ plane), most interpolants are truly spatial curves.

\footnotetext{
${ }^{2}$ Any fixed value of $\phi_{1}$ gives equivalent results. In [9] authors choose $\phi_{1}=-\pi / 2$.
} 


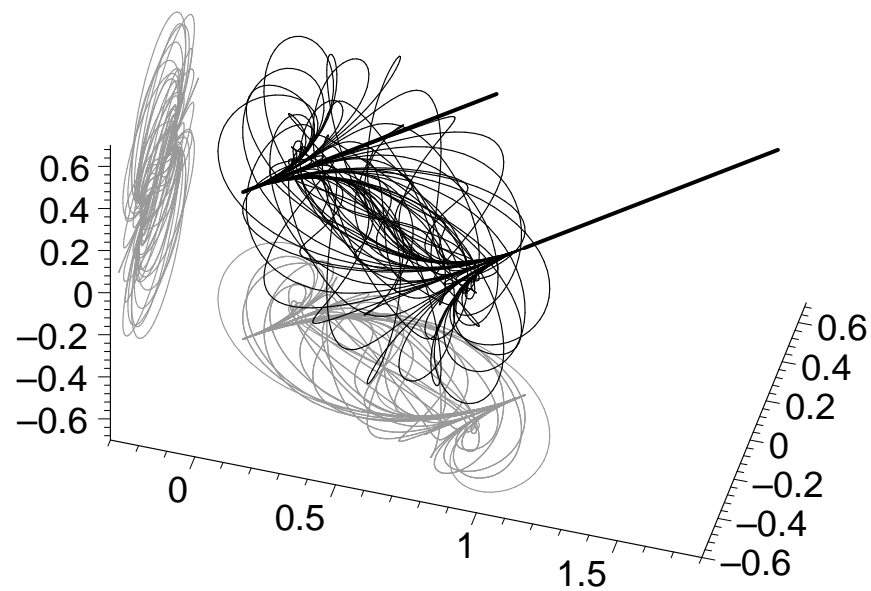

Fig. 1. The system of space PH quintic interpolants to given data. 64 representatives are plotted, along with their projections into the $x y$ and $y z$ planes (gray lines). The end-point tangent vectors are also shown, scaled by $1 / 4$.

\subsection{Invariance of interpolants}

For any given Hermite data $\mathbf{p}_{0}, \mathbf{p}_{1}, \mathbf{t}_{0}, \mathbf{t}_{1}$, the system $\left\{\mathbf{p}_{\phi_{0}, \phi_{2}}(t) \mid \phi_{0}, \phi_{2} \in[0,2 \pi)\right\}$ represents all PH Hermite interpolants. Therefore, it is invariant [10] with respect to orthogonal transformations (including reflections).

More precisely, if we apply an orthogonal transformation $\Xi$ to the Hermite data, we get modified data $\tilde{\mathbf{p}}_{0}, \tilde{\mathbf{p}}_{1}, \tilde{\mathbf{t}}_{0}, \tilde{\mathbf{t}}_{1}$. The associated systems of interpolants then satisfy

$$
\left\{\tilde{\mathbf{p}}_{\tilde{\phi}_{0}, \tilde{\phi}_{2}}(t) \mid \tilde{\phi}_{0}, \tilde{\phi}_{2} \in[0,2 \pi)\right\}=\Xi\left(\left\{\mathbf{p}_{\phi_{0}, \phi_{2}}(t) \mid \phi_{0}, \phi_{2} \in[0,2 \pi)\right\}\right) .
$$

On the other hand, this transformation does not preserve the parameterization of the solutions: In general

$$
\tilde{\mathbf{p}}_{\tilde{\phi}_{0}, \tilde{\phi}_{2}}(t)=\Xi\left(\mathbf{p}_{\phi_{0}, \phi_{2}}(t)\right)
$$

is not valid for $\tilde{\phi}_{0}=\phi_{0}, \tilde{\phi}_{2}=\phi_{2}$.

The relation between $\phi_{0}, \phi_{2}$ and $\tilde{\phi}_{0}, \tilde{\phi}_{2}$ ensuring (20) is rather complicated [9]. Still, it can be formulated easily in the following cases.

Lemma 3. For any $\phi_{0}, \phi_{2}$ :

1. If $\Xi$ is a rotation about the $\mathbf{i}$-axis, then

$$
\tilde{\mathbf{p}}_{\phi_{0}, \phi_{2}}(t)=\Xi\left(\mathbf{p}_{\phi_{0}, \phi_{2}}(t)\right) \text {. }
$$

2. If $\Xi$ is a reflection with respect to a plane containing the $\mathbf{i}$-axis, then

$$
\tilde{\mathbf{p}}_{\phi_{0}, \phi_{2}}(t)=\Xi\left(\mathbf{p}_{-\phi_{0},-\phi_{2}}(t)\right) \text {. }
$$


Proof. Consider fixed values of $\phi_{0}, \phi_{2}$ and let $\mathcal{A}_{0}, \mathcal{A}_{1}, \mathcal{A}_{2}$ denote the control points of the preimage for some data $\mathbf{p}_{0}, \mathbf{p}_{1}, \mathbf{t}_{0}, \mathbf{t}_{1}$ and $\tilde{\mathcal{A}}_{0}, \tilde{\mathcal{A}}_{1}, \tilde{\mathcal{A}}_{2}$ for the transformed data $\tilde{\mathbf{p}}_{0}=\Xi\left(\mathbf{p}_{0}\right), \tilde{\mathbf{p}}_{1}=\Xi\left(\mathbf{p}_{1}\right), \tilde{\mathbf{t}}_{0}=\Xi\left(\mathbf{t}_{0}\right), \tilde{\mathbf{t}}_{1}=\Xi\left(\mathbf{t}_{1}\right)$.

1) Any rotation can be expressed using formula (6). If $\Xi$ is rotation about the $\mathbf{i}$-axis through angle $\theta$, then for any vector $\mathbf{c}$ we have

$$
\Xi(\mathbf{c})=\mathcal{Q}\left(\frac{\theta}{2}\right) \mathbf{c} \mathcal{Q}\left(-\frac{\theta}{2}\right) .
$$

The right-hand side of the previous equation allows to extend the transformation $\Xi$ from pure quaternions to all quaternions. Because of the form of the equations (9) and the fact that $\mathcal{Q}(\phi) \mathcal{Q}\left(\frac{\theta}{2}\right)=\mathcal{Q}\left(\frac{\theta}{2}\right) \mathcal{Q}(\phi)$,

$$
\tilde{\mathcal{A}}_{0}=\Xi\left(\mathcal{A}_{0}\right) \text {, and } \tilde{\mathcal{A}}_{2}=\Xi\left(\mathcal{A}_{2}\right) \text {. }
$$

Now using (21) and (22)

$$
\left(\tilde{\mathcal{A}}_{0} \mathbf{i} \tilde{\mathcal{A}}_{2}^{*}+\tilde{\mathcal{A}}_{2} \mathbf{i} \tilde{\mathcal{A}}_{0}^{*}\right)=\Xi\left(\mathcal{A}_{0} \mathbf{i} \mathcal{A}_{2}^{*}+\mathcal{A}_{2} \mathbf{i} \mathcal{A}_{0}^{*}\right)
$$

Consequently, in step 2, the right-hand side of equation (16) for the transformed data is equal to the transformed right-hand side of this equation for the original data. Hence,

$$
\tilde{\mathcal{A}}_{1}=\Xi\left(\mathcal{A}_{1}\right) \text { and thus for the whole preimage curve } \tilde{\mathcal{A}}(t)=\Xi(\mathcal{A}(t)) .
$$

Finally

$$
\tilde{\mathbf{p}}(\tau)=\tilde{\mathbf{p}}_{0}+\int_{0}^{\tau} \tilde{\mathcal{A}}(t) \mathbf{i} \tilde{\mathcal{A}}^{*}(t) \mathrm{d} t=\Xi\left(\mathbf{p}_{0}\right)+\int_{0}^{\tau} \Xi\left(\mathcal{A}(t) \mathbf{i} \mathcal{A}^{*}(t)\right) \mathrm{d} t=\Xi(\mathbf{p}(\tau)) .
$$

2) Due to the first part of the lemma, it suffices to consider only the reflection $\Xi_{\mathbf{k}}$ with respect to the $\mathbf{i}, \mathbf{j}$ plane. Indeed, any other reflection with respect to a plane containing the $\mathbf{i}$ axis can be obtained as a composition of $\Xi_{\mathbf{k}}$ and two rotations about the $\mathbf{i}$ axis.

$\Xi_{\mathbf{k}}$ can be extended to all quaternions setting

$$
\Xi_{\mathbf{k}}(a+b \mathbf{i}+c \mathbf{j}+d \mathbf{k})=-a+b \mathbf{i}+c \mathbf{j}-d \mathbf{k}
$$

A direct computation confirms, that formulas (22)-(25) are still valid, if the control points $\tilde{\mathcal{A}}_{i}$ are constructed with parameters $-\phi_{0},-\phi_{2}$, while the control points $\mathcal{A}_{i}$ are constructed with the parameters $\phi_{0}, \phi_{2}$.

A fully invariant parameterization of interpolants is obtained by considering a standard position.

Definition 1. The $C^{1}$ spatial Hermite data are said to be in a standard position, if $\mathbf{t}_{0}+\mathbf{t}_{1}$ is a positive multiple of $\mathbf{i}$, and $\mathbf{p}_{0}=0$.

From now on, we will use the following parameterization of the system of interpolants. 
Definition 2. The system of interpolants is parameterized by the two parameters $\phi_{0}, \phi_{2}$, as follows. First we transform data to a standard position, where we construct the interpolants $\mathbf{p}_{\phi_{0}, \phi_{2}}(t)$, as described before. Finally, we transform the solution back to the original position.

Note that parameterization is well-defined, since Lemma 3 ensures that the particular choice of a standard position (which may vary by a rotation about $\mathbf{i}$ axis) does not matter.

Theorem 1. The parameterization of the solutions, according to Definition 2, is invariant with respect to rigid body motions (special orthogonal transformations), whereas reflections change the signs of both parameters. Consequently, the solution $\mathbf{p}_{0,0}(t)$ is invariant with respect to all orthogonal transformations.

The proof results from Lemma 3.

In addition, the parameterization of the solutions is symmetric in the following sense.

Theorem 2. Let $\mathbf{p}_{\phi_{1}, \phi_{2}}(t)$ be the interpolants of data $\mathbf{p}_{0}, \mathbf{p}_{1}, \mathbf{t}_{0}, \mathbf{t}_{1}$ and $\overline{\mathbf{p}}_{\phi_{0}, \phi_{2}}(t)$ the interpolants of the "reversed" data $\overline{\mathbf{p}}_{0}=\mathbf{p}_{1}, \overline{\mathbf{p}}_{1}=\mathbf{p}_{0}, \overline{\mathbf{t}}_{0}=-\mathbf{t}_{1}, \overline{\mathbf{t}}_{1}=-\mathbf{t}_{0}$. Then for any $\phi_{0}, \phi_{2}$

$$
\overline{\mathbf{p}}_{\phi_{0}, \phi_{2}}(1-t)=\mathbf{p}_{-\phi_{2},-\phi_{0}}(t) .
$$

Proof. Suppose that the given data to be in a standard position, i.e. $\mathbf{t}_{0}+\mathbf{t}_{1}$ is a positive multiple of $\mathbf{i}$ and $\mathbf{p}_{0}=0$. The reversed data can be transformed into a new standard position by the translation of the vector $-\mathbf{p}_{1}$ and the rotation given as composition of the the symmetry $S: \mathbf{c} \rightarrow-\mathbf{c}$ and reflection $\Xi_{\mathbf{k}}(26)$. In fact, $S$ already transforms the reversed data into a standard position, but it is not a rotation $(\operatorname{det}(S)=-1)$.

The standard position associated with the reversed data differs from the original data only by swapping $\mathbf{t}_{0}$ and $\mathbf{t}_{1}$ and by the reflection $\Xi_{\mathbf{k}}$. The theorem then follows from the symmetry of the system of equations (15)-(16) with respect to $\mathcal{A}_{0}$ and $\mathcal{A}_{2}$, and from the second part of Lemma 3 .

\section{Qualitative analysis of the interpolants}

In this section we give a qualitative analysis of the system of $\mathrm{PH}$ quintic interpolants, in order to identify the 'best' values of the parameters $\phi_{0}, \phi_{2}$. These parameters yield the interpolants suitable for applications.

\subsection{Asymptotic behavior}

In order to fix the free parameters $\phi_{0}, \phi_{2}$, we will now study the asymptotic behavior of the solutions $\mathbf{p}_{\phi_{0}, \phi_{2}}(t)$. More precisely, we assume that the $C^{1}$ Hermite data are taken from a small segment of an analytical curve, and we investigate the asymptotic behavior of the solutions for decreasing step-size. 
We assume that the curve is given by its Taylor expansion. Without loss of generality,

$$
\mathbf{C}(T)=\left(T+\sum_{i=2}^{\infty} \frac{x_{i}}{i !} T^{i}, \sum_{i=2}^{\infty} \frac{y_{i}}{i !} T^{i}, \sum_{i=2}^{\infty} \frac{z_{i}}{i !} T^{i}\right)^{\top}
$$

with arbitrary coefficients $x_{2}, x_{3}, \ldots, y_{2}, y_{3}, \ldots$ and $z_{2}, z_{3}, \ldots$

For any step-size $h$, we pick the segment $\mathbf{c}(t)=\mathbf{C}(h t), t \in[0,1]$. This segment has the expansion

$$
\mathbf{c}(t)=\left(t h+\sum_{i=2}^{\infty} \frac{x_{i}}{i !} t^{i} h^{i}, \sum_{i=2}^{\infty} \frac{y_{i}}{i !} t^{i} h^{i}, \sum_{i=2}^{\infty} \frac{z_{i}}{i !} t^{i} h^{i}\right)^{\top} .
$$

Now we interpolate the $C^{1}$ Hermite boundary data at the points $\mathbf{c}(0)=\mathbf{C}(0)$ and $\mathbf{c}(1)=\mathbf{C}(h)$. Depending on the interval size $h$, different PH curves interpolating the data behave as described in the following Theorem.

Theorem 3. The error of the PH interpolation

$$
\max _{t \in[0,1]}\left\|\mathbf{c}(t)-\mathbf{p}_{\phi_{0}, \phi_{2}}(t)\right\|
$$

converges to 0 as $\mathcal{O}\left(h^{4}\right)$ if and only if $\phi_{0}=\phi_{2}=0$. Otherwise it converges to 0 only as $\mathcal{O}\left(h^{1}\right)$.

Proof. The proof consists in evaluating power series of all quantities occurring in the interpolation process with respect to the step size $h$. This can be done by a suitable computer algebra tool. Due to the space limitation and the complexity of the expressions, we show only the leading terms of certain quantities, in order to illustrate the idea of our approach.

First, we derive the Taylor expansions of the Hermite boundary data at $t=0$ and $t=1$ of the curve (29),

$$
\begin{aligned}
& \mathbf{p}_{0}=\left(\begin{array}{l}
0 \\
0 \\
0
\end{array}\right) \mathbf{p}_{1}=\left(\begin{array}{l}
h+\frac{1}{2} x_{2} h^{2}+\frac{1}{6} x_{3} h^{3}+\ldots \\
\frac{1}{2} y_{2} h^{2}+\frac{1}{6} y_{3} h^{3}+\ldots \\
\frac{1}{2} z_{2} h^{2}+\frac{1}{6} z_{3} h^{3}+\ldots
\end{array}\right) \\
& \mathbf{t}_{0}=\left(\begin{array}{l}
h \\
0 \\
0
\end{array}\right) \mathbf{t}_{1}=\left(\begin{array}{l}
h+x_{2} h^{2}+\frac{1}{2} x_{3} h^{3}+\ldots \\
y_{2} h^{2}+\frac{1}{2} y_{3} h^{3}+\ldots \\
z_{2} h^{2}+\frac{1}{2} z_{3} h^{3}+\ldots
\end{array}\right) .
\end{aligned}
$$

This data can be transformed into a standard position by a rotation

$$
U=\left(\begin{array}{lll}
1-\frac{y_{2}{ }^{2}+z_{2}{ }^{2}}{8} h^{2}+\ldots & \frac{y_{2}}{2} h+\frac{y_{3}-y_{2} x_{2}}{4} h^{2}+\ldots & \frac{z_{2}}{2} h+\frac{z_{3}-z_{2} x_{2}}{4} h^{2}+\ldots \\
-\frac{y_{2}}{2} h-\frac{y_{3}-y_{2} x_{2}}{4} h^{2}+\ldots & 1-\frac{y_{2}^{2}}{8} h^{2}+\ldots & 0 \\
-\frac{z_{2}}{2} h-\frac{z_{3}-z_{2} x_{2}}{4} h^{2}+\ldots & -\frac{z_{2} y_{2}}{4} h^{2}+\ldots & 1-\frac{z_{2}{ }^{2}}{8} h^{2}+\ldots
\end{array}\right) .
$$


Then we compute the Taylor expansions of the control points of the preimage for the transformed data $U\left(\mathbf{p}_{0}\right), U\left(\mathbf{p}_{1}\right), U\left(\mathbf{t}_{0}\right), U\left(\mathbf{t}_{1}\right)$. Using (9) we obtain

$$
\begin{aligned}
\mathcal{A}_{0}\left(\phi_{0}\right)= & -\sqrt{h}\left[\sin \phi_{0}+\ldots\right]+\sqrt{h}\left[\cos \phi_{0}+\ldots\right] \mathbf{i}-\sqrt{h}\left[\frac{y_{2} \cos \phi_{0}+z_{2} \sin \phi_{0}}{4} h+\ldots\right] \mathbf{j} \\
& -\sqrt{h}\left[\frac{z_{2} \cos \phi_{0}-y_{2} \sin \phi_{0}}{4} h+\ldots\right] \mathbf{k} \\
\mathcal{A}_{2}\left(\phi_{2}\right)= & -\sqrt{h}\left[\sin \phi_{2}+\frac{x_{2} \sin \phi_{2}}{2} h+\ldots\right]+\sqrt{h}\left[\cos \phi_{2}+\frac{x_{2} \cos \phi_{2}}{2} h+\ldots\right] \mathbf{i} \\
& +\sqrt{h}\left[\frac{y_{2} \cos \phi_{2}+z_{2} \sin \phi_{2}}{4} h+\ldots\right] \mathbf{j}+\sqrt{h}\left[\frac{z_{2} \cos \phi_{2}-y_{2} \sin \phi_{2}}{4} h+\ldots\right] \mathbf{k}
\end{aligned}
$$

(step 1). In step 2 (with the fixed choice $\phi_{1}=0$ ) we obtain the expansion of $\mathcal{A}_{1}$, involving both $\phi_{0}, \phi_{2}$. We omit it here since even the leading terms are rather complicated.

Finally we are able to express the Taylor expansion of the $\mathrm{PH}$ interpolant $\mathbf{p}_{\phi_{1}, \phi_{2}}(t)$, which is again a long expression. Still, the leading term of its $x$ component is equal to

$$
\begin{aligned}
& {\left[t+\frac{1}{2}\left(\cos \left(\phi_{0}\right) \sqrt{10 \cos \left(\phi_{2}-\phi_{0}\right)+90}-3 \cos \left(\phi_{2}-\phi_{0}\right)-7\right) t^{2}\right.} \\
& -\frac{1}{2}\left(\left[3 \cos \left(\phi_{0}\right)+\cos \left(\phi_{2}\right)\right] \sqrt{10 \cos \left(\phi_{2}-\phi_{0}\right)+90}-12 \cos \left(\phi_{2}-\phi_{0}\right)-28\right) t^{3} \\
& +\frac{1}{2}\left(\left[3 \cos \left(\phi_{0}\right)+2 \cos \left(\phi_{2}\right)\right] \sqrt{10 \cos \left(\phi_{2}-\phi_{0}\right)+90}-15 \cos \left(\phi_{2}-\phi_{0}\right)-35\right) t^{4} \\
& \left.-\frac{1}{2}\left(\left[\cos \left(\phi_{0}\right)+\cos \left(\phi_{2}\right)\right] \sqrt{10 \cos \left(\phi_{2}-\phi_{0}\right)+90}-6 \cos \left(\phi_{2}-\phi_{0}\right)-14\right) t^{5}\right] h .
\end{aligned}
$$

Comparing this series with (29), we see that the coefficients at $t^{2}, t^{3}, t^{4}, t^{5}$ in (33) must be zero if the interpolant $\mathbf{p}_{\phi_{1}, \phi_{2}}(t)$ should match the the shape of $\mathbf{c}(t)$ and the error (30) should converge to 0 faster then $\mathcal{O}(h)$. Solving the system of trigonometric equations it can be shown, that this is achieved if and only if $\phi_{0}=\phi_{2}=0$. Using these values, the Taylor expansion of $\mathbf{p}_{0,0}(t)$ simplifies enormously, and matches the Taylor expansion of $\mathbf{c}(t)$ up to $h^{3}$.

Remark 1. Though the proof Theorem 3 seems to be complicated, it is in fact a straightforward computation. It would be interesting to proof a more general result stating that any $C^{1}$ Hermite interpolation satisfying certain conditions always leads to approximation order 4 , as it is the case for Hermite interpolation by Bézier cubics and $\mathrm{PH}$ quintics $\mathbf{p}_{0,0}(t)$. This may be a subject of future research.

Remark 2. In Theorem 3 we considered only constant values of $\phi_{0}$ and $\phi_{2}$, which do not depend on the the step size $h$. It may also be interesting to choose these parameters depending on the step-size. Thus $\phi_{0}(h), \phi_{2}(h)$ would be functions of $h$. In this more general setting we still can study the asymptotic behavior. It is only necessary to replace the constants $\phi_{0}, \phi_{2}$ by abstract Taylor series of the functions $\phi_{0}(h), \phi_{2}(h)$. We obtained the following result about the approximation order.

The error of the $\mathrm{PH}$ interpolation

$$
\max _{t \in[0,1]}\left\|\mathbf{c}(t)-\mathbf{p}_{\phi_{0}(h), \phi_{2}(h)}(t)\right\|
$$

is equal to $\mathcal{O}\left(h^{4}\right)$ if and only if 
1. $\lim _{h \rightarrow 0} \phi_{1}(h)=\lim _{h \rightarrow 0} \phi_{2}(h)=0$ and

2. $\phi_{2}^{\prime}(0)=-\phi_{0}^{\prime}(0)$.

If only the first condition holds, the error of the approximation equals $\mathcal{O}\left(h^{3}\right)$. If 1 . is not satisfied, then the error equals $\mathcal{O}\left(h^{1}\right)$.

Remark 3. The interpolant $\mathbf{p}_{0,0}(t)$ has the following interesting property. If $\phi_{0}=$ $\phi_{1}=\phi_{2}=0$, then the control points $\mathcal{A}_{0}, \mathcal{A}_{1}, \mathcal{A}_{2}$ are pure quaternions, and therefore the whole preimage $\mathcal{A}(t)$ is pure-quaternion valued. This corresponds to setting $u(t)=0$ in the representation formula (11), which then becomes

$$
\begin{aligned}
x^{\prime}(t) & =v^{2}(t)-p^{2}(t)-q^{2}(t), \\
y^{\prime}(t) & =2 v(t) p(t), \\
z^{\prime}(t) & =2 v(t) q(t), \\
\sigma(t) & =v^{2}(t)+p^{2}(t)+q^{2}(t) .
\end{aligned}
$$

This incomplete description of PH curves was used first in [5]. The optimal solution $\mathbf{p}_{0,0}(t)$ is therefore given by transforming the curve into standard position and constructing one of the interpolants of the form (34).

\subsection{Preservation of planarity}

As a natural question, one may ask which interpolants $\mathbf{p}_{\phi_{0}, \phi_{2}}$ are planar for planar input data.

$C^{1}$ Hermite interpolation by $\mathrm{PH}$ quintics in the plane is well understood. It has been shown, that for any non-degenerated planar data there are four planar $\mathrm{PH}$ quintic interpolants [6], which have been in [20] labeled as $(++),(+-)$, $(-+)$ and $(--)$. In the following new result we identify them among the two parameter family of spatial interpolants.

Theorem 4. For any input data $\mathbf{p}_{0}, \mathbf{p}_{1}, \mathbf{t}_{0}, \mathbf{t}_{1}$ lying in a plane, the four interpolants $\mathbf{p}_{0,0}(t), \mathbf{p}_{0, \pi}(t), \mathbf{p}_{\pi, 0}(t), \mathbf{p}_{\pi, \pi}(t)$ are planar. They can be identified in the following way with the interpolants in [20]:

$$
\mathbf{p}_{0,0}(t)=(++), \mathbf{p}_{0, \pi}(t)=(+-), \mathbf{p}_{\pi, 0}(t)=(-+), \text { and } \mathbf{p}_{\pi, \pi}(t)=(--) .
$$

Proof. We suppose that the input data lie in the $\mathbf{i}, \mathbf{j}$ plane, which we will denote with $\mathbb{Q}_{\mathrm{ij}}$. We define on the quaternions the commutative multiplication

$$
\mathcal{U} \star \mathcal{V}:=\frac{1}{2}\left(\mathcal{U} \mathbf{i} \mathcal{V}^{*}+\mathcal{V} \mathbf{i} \mathcal{U}^{*}\right)
$$

One can verify directly, that $\left(\mathbb{Q}_{\mathrm{ij}}, \star\right) \simeq \mathbb{C}$. Under this isomorphism, the equations (15)-(16) become the complex equations characterizing PH interpolation in plane (equations (3)-(4) of [20]).

Obviously, for the choice $\phi_{0}=0$, resp. $\phi_{0}=\pi$, the control point $\mathcal{A}_{0}$ is in $\mathbb{Q}_{\mathrm{ij}}$ and correspond to the complex square root of $\mathbf{t}_{0}$ with positive, resp. negative real part, which is precisely the principle of the labeling used in [20]. Similarly for $\mathcal{A}_{2}$ and $\phi_{2}$. Then also $\mathcal{A}_{1} \in \mathbb{Q}_{\mathbf{i j}}$ and the correspondence (35) holds. 




Fig. 2. Planar interpolants of Hermite data (19). The interpolant $\mathbf{p}_{0,0}$ is plotted in bold. Projections to $x y$ and $y z$ plane are plotted in grey.

Figure 2 shows the four planar interpolants $\mathbf{p}_{0,0}(t), \mathbf{p}_{0, \pi}(t), \mathbf{p}_{\pi, 0}(t), \mathbf{p}_{\pi, \pi}(t)$ to the planar data (19) (see also the previous figure). Note that the projections (grey) into the $y z$ plane collapse into line segments.

\section{Applications}

We apply the previous results in order to design an algorithm converting any analytical curve into a piecewise $\mathrm{PH}$ quintic curve. This conversion is then used for approximation of pipe surfaces.

\subsection{Conversion of analytical curves}

The result described in Theorem 3 allows us to design an algorithm for the conversion of any analytical curve into a piecewise $\mathrm{PH}$ curve. Let the parameter domain of the analytical curve be $[0,1]$. We split this interval into the $2^{n}$ subintervals $\left[\frac{i}{2 n}, \frac{i+1}{2 n}\right], i=0 . .2 n-1$. For each subinterval, we construct the $\mathrm{PH}$ Hermite interpolant $\mathbf{p}_{0,0}(t)$ and obtain a $C^{1}$ continuous piecewise $\mathrm{PH}$ curve of degree 5 . If the error from the original analytical curve is not sufficiently small, we continue the subdivision. Due to the Proposition 3, the error will converge to 0 as $\mathcal{O}\left(\frac{1}{16^{n}}\right)$ under subdivision.

The relatively high rate of convergence is demonstrated by the following example. Figure 3 shows the segment of the analytical curve

$$
\mathbf{c}(t)=\left(1.5 \sin (7.2 t), \cos (9 t), e^{\cos (1.8 t)}\right)^{\top}, t \in[0,1] .
$$

We construct the PH Hermite interpolant for the whole segment and the piecewise $\mathrm{PH}$ interpolants obtained after splitting the parameter into $2,4,8, \ldots, 512$ 

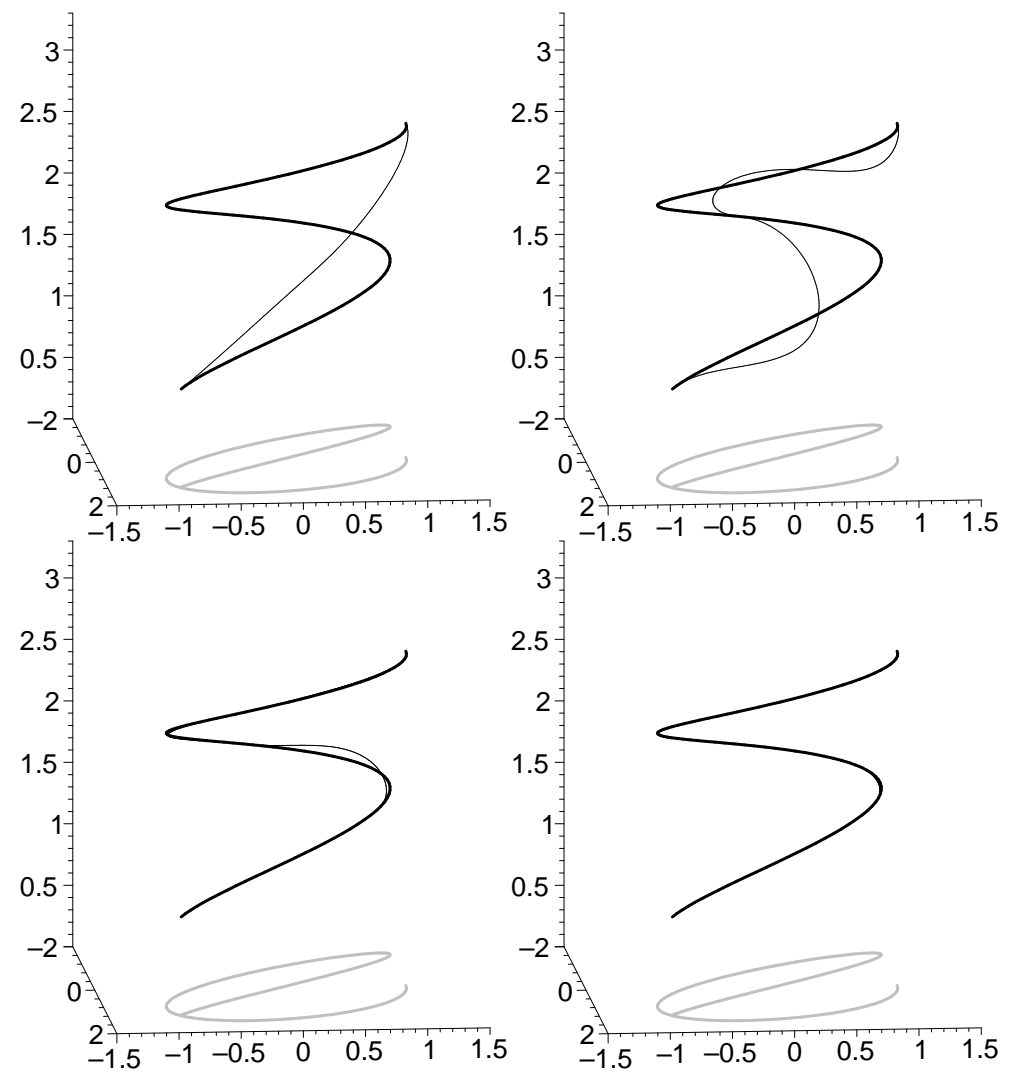

Fig. 3. Approximate conversion of an analytical curve (bold line) via $C^{1}$ Hermite interpolation by $\mathrm{PH}$ curves, obtained after splitting the parameter domain into 1, 2, 4 and 8 segments. The difference between the curves is almost invisible in the last case. In addition to the curve its projection into $x y$ plane is plotted (gray line).

subintervals. The maximal approximation error and its improvement (ratio) in each step are shown in Table 1.

Clearly, instead of the simple uniform subdivision, using an adaptive subdivision scheme would reduce the number of segments.

\subsection{Approximation of pipe surfaces}

PH curves possess a simple low degree rational adapted frame, which has been called the Euler-Rodrigues frame in [3]. Based on this construction, Farouki proposed a rational approximation of the rotation minimizing frame for any space $\mathrm{PH}$ curve [13]. 


\begin{tabular}{|c|l|l||c|l|l|}
\hline \#Segments & Error & Ratio & \#Segments & Error & Ratio \\
\hline \hline 1 & 2.429 & & 32 & $1.94110^{-4}$ & $10.67 \times$ \\
\hline 2 & 1.384 & $1.76 \times$ & 64 & $1.33710^{-5}$ & $14.52 \times$ \\
\hline 4 & $1.55310^{-1}$ & $8.91 \times$ & 128 & $8.52310^{-7}$ & $15.68 \times$ \\
\hline 8 & $2.39910^{-2}$ & $6.48 \times$ & 256 & $5.37610^{-8}$ & $15.85 \times$ \\
\hline 16 & $2.07010^{-3}$ & $11.59 \times$ & 512 & $3.36110^{-9}$ & $16.00 \times$ \\
\hline
\end{tabular}

Table 1. Error of piecewise quintic PH approximation via Hermite interpolation.

Both frames can be used for the approximation of pipe surfaces, or - more generally - of sweep surfaces. First we convert a given analytical curve into a piecewise $\mathrm{PH}$ curve using the algorithm of section 5.1 and then we construct a pipe surface for this $\mathrm{PH}$ curve.

The approximation error can be defined in the following way. The exact pipe surface with radius $r$ can be understood as a union of circles $S(t)$, with centers $\mathbf{c}(t)$ and lying in the normal plane of $\mathbf{c}$ at point $t$. Similarly, our approximation of the pipe surface can be seen as collection of the circles $\tilde{S}(t)$.

For each parameter value $t$, we define $E(t)$ as the Hausdorff distance of the circles $S(t)$ and $\tilde{S}(t)$. Then the global error of the approximation is defined as

$$
E=\max _{t \in[0,1]} E(t) .
$$

Theorem 5. The approximation error $E$ behaves as $\mathcal{O}\left(h^{3}\right)$ for $h \rightarrow 0$.

Proof. Using the triangle inequality, the Hausdorff distance between circles $S(t)$ and $\tilde{S}(t)$ can be bounded as

$$
E(t) \leq r\left\|\frac{\mathbf{c}^{\prime}(t)}{\left\|\mathbf{c}^{\prime}(t)\right\|}-\frac{\mathbf{p}_{0,0}^{\prime}(t)}{\left\|\mathbf{p}_{0,0}^{\prime}(t)\right\|}\right\|+\left\|\mathbf{c}(t)-\mathbf{p}_{0,0}(t)\right\|,
$$

where the first term represents the Hausdorff distance between two circles with a common center and the second one is the distance between the centers. According to Theorem 3

$$
\max _{t \in[0,1]}\left\|\mathbf{c}(t)-\mathbf{p}_{0,0}(t)\right\|=\mathcal{O}\left(h^{4}\right) .
$$

In a similar way one can prove that

$$
\max _{t \in[0,1]}\left\|\frac{\mathbf{c}^{\prime}(t)}{\left\|\mathbf{c}^{\prime}(t)\right\|}-\frac{\mathbf{p}_{0,0}^{\prime}(t)}{\left\|\mathbf{p}_{0,0}^{\prime}(t)\right\|}\right\|=\mathcal{O}\left(h^{3}\right),
$$

which concludes the proof.

As an example, Figure 4 shows an approximation of a pipe surface associated with the curve (37), constructed using a piecewise $\mathrm{PH}$ curve composed of 8 segments (see last figure of Fig. 3). 


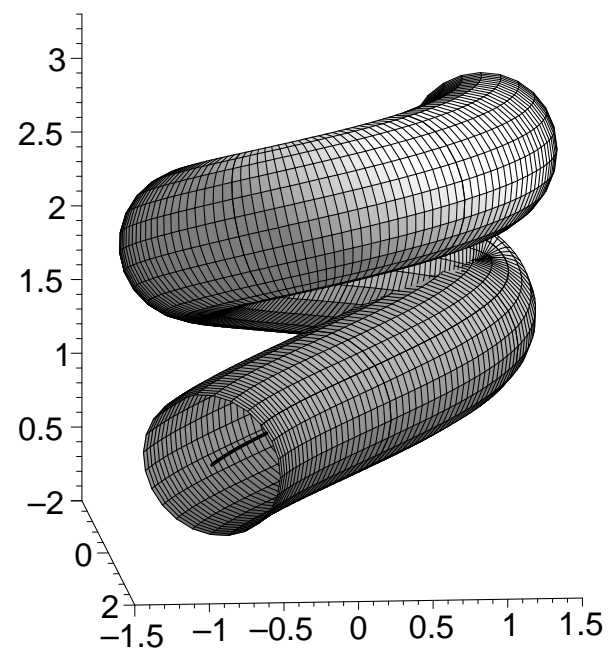

Fig. 4. Approximation of a pipe surface.

\section{Conclusion}

Starting from previous results about quintic PH curves in two and three dimensions $[9,20]$, we analyzed the system of solutions and identified one of them, which is suitable for applications. More precisely, it is invariant under orthogonal transformations (rigid body transformations and reflections), preserves planarity, it is invariant with respect to reversion of the parameter interval, and it has the optimum approximation order. We used this solution for approximately converting general curves into $\mathrm{PH}$ form and for approximation of pipe surfaces.

It should be noted that choosing this solution leads to a significant improvement of the approximation order. In order to achieve the same error with other solutions, 8 times as many intervals would be needed. Also, the shape might be less pleasing.

As a matter of future work, we will investigate the problem of $C^{2}$ Hermite interpolation in three-dimensional space. According to our experience in the planar case, the use of geometric Hermite data (points, tangent directions, curvatures) always produces problems with specific points, such as inflections, while these difficulties are not present for analytical data (points, first and second derivatives). Consequently, the use of analytical data seems to be more appropriate.

Acknowledgment. The first author was supported through grant P17387-N12 of the Austrian Science Fund (FWF).

\section{References}

1. M.-H. Ahn, G.-I. Kim and C.-N. Lee, Geometry of root-related parameters of PH curves. Appl. Math. Lett. 16 (2003), no. 1, 49-57. 
2. H.I. Choi, D.S. Lee and H.P. Moon (2002), Clifford algebra, spin representation, and rational parameterization of curves and surfaces. Adv. Comput. Math. 17, 5-48.

3. H.I. Choi, C.Y. Han, Euler Rodrigues frames on spatial Pythagorean-hodograph curves, Comput. Aided Geom. Design 19 (2002) 603-620.

4. R. Dietz, J. Hoschek, B. Jüttler, An algebraic approach to curves and surfaces on the sphere and on other quadrics, Comp. Aided Geom. Design 10 (1993), 211-229.

5. R.T. Farouki and T. Sakkalis, Pythagorean-hodograph space curves, Adv. Comput. Math. 2 (1994) 41-66.

6. R.T. Farouki and C.A. Neff (1995), Hermite interpolation by Pythagoreanhodograph quintics. Math. Comput. 64, 1589-1609.

7. R. T. Farouki and K. Saitou and Y-F. Tsai (1998), Least-squares tool path approximation with Pythagorean-hodograph curves for high-speed CNC machining. The Mathematics of Surfaces VIII, Information Geometers, Winchester, 245-264.

8. R.T. Farouki, B.K. Kuspa, C. Manni, and A. Sestini (2001), Efficient solution of the complex quadratic tridiagonal system for $C^{2} \mathrm{PH}$ quintic splines, Numer. Alg. $27,35-60$.

9. R. T. Farouki, M. al-Kandari and T. Sakkalis (2002), Hermite interpolation by rotation-invariant spatial Pythagorean-hodograph curves., Adv. Comput. Math. 17, 369-383.

10. R. T. Farouki, M. al-Kandari and T. Sakkalis (2002), Structural invariance of spatial Pythagorean hodographs, Comp. Aided Geom. Design 19, 395-407.

11. R.T. Farouki (2002), Pythagorean hodograph curves, in G. Farin, J. Hoschek and M.-S. Kim (eds.), Handbook of Computer Aided Geometric Design, North-Holland, Amsterdam, 405-427.

12. R.T. Farouki, C. Manni, A. Sestini (2003), Spatial $C^{2}$ PH quintic splines. Curve and surface design (Saint-Malo, 2002), 147-156, Mod. Methods Math., Nashboro Press.

13. R.T. Farouki, C. Y. Han (2003), Rational approximation schemes for rotationminimizing frames on Pythagorean- hodograph curves, Computer Aided Geometric Design 20, 435-454.

14. R.T. Farouki, C.Y. Han, C. Manni, A. Sestini (2004), Characterization and construction of helical polynomial space curves, J. Comput. Appl. Math. 162, no. 2, 365-392.

15. R. Feichtinger (2005), Hermite-Interpolation mit PH-Quintiken, Diplomarbeit (MSc. thesis), Johannes Kepler University, Linz, Austria, in progress.

16. J. Hoschek and D. Lasser (1996), Fundamentals of Computer Aided Geometric Design, AK Peters, Wellesley MA.

17. B. Jüttler and C. Mäurer (1999), Cubic Pythagorean Hodograph Spline Curves and Applications to Sweep Surface Modeling, Comp.-Aided Design 31, 73-83.

18. B. Jüttler (2001), Hermite interpolation by Pythagorean hodograph curves of degree seven. Math. Comp. 70, 1089-1111.

19. J.B. Kuipers (1999), Quaternions and rotation sequences. Princeton University Press.

20. H.P. Moon, R.T. Farouki and H.I. Choi (2001), Construction and shape analysis of PH quintic Hermite interpolants, Comp. Aided Geom. Design 18, 93-115.

21. Z. Š́r and B. Jüttler (2005), Constructing acceleration continuous tool paths using pythagorean hodograph curves. Mech. Mach. Theory. In press.

22. D.S. Meek and D.J. Walton (1997), Geometric Hermite interpolation with Tschirnhausen cubics, Journal of Computational and Applied Mathematics 81, 299-309.

23. D.J. Walton and D.S. Meek (2004), A generalisation of the Pythagorean hodograph quintic spiral. J. Comput. Appl. Math. 172, no. 2, 271-287. 